\title{
THE RELATION BETWEEN LINEAR EXTENT AND VELOCITY IN DRAWING MOVEMENTS
}

\author{
P. VIVIANI* and G. MCCOLlum ${ }^{*}$ \\ Laboratoire de Physiologie Neurosensorielle, CNRS, Paris, France
}

\begin{abstract}
The speed of execution of complex movements depends on both the local, differential properties of the trajectory and on some of its more global metric parameters. The effects of these global factors were studied in free, writing-like movements with either piece-wise constant, or regularly changing curvature. It is demonstrated that the tangential velocity of the pen's tip is tightly correlated, through a power function, with the total linear extent of the trajectory (perimeter). Thus, a strong tendency exists to keep the execution time of these complex trajectories independent of the movement size (isochrony). Furthermore, it is shown that the average tangential velocity over identifiable segments of the trajectory also depends on the corresponding average curvature. The implications of these results vis-à-vis the central representation and planning of movements are discussed.
\end{abstract}

A very general compensatory mechanism called the Isochrony Principle ${ }^{7,12}$ seems to apply to many areas of motor control. In the broadest qualitative terms, the principle states that the velocity of voluntary movements increases with the extent of the movement, thus keeping execution time approximately constant. ${ }^{3.10}$ Ilowever, such compensatory behavior emerges in motor performances as sharply different as manual pointing, ${ }^{4}$ stroking ${ }^{1.8}$ handwriting, ${ }^{5}{ }^{5.11}$ head rotations ${ }^{4,5}$ and saccadic eye movements, ${ }^{6,9}$ taking different quantitative forms according to both the specific motor system involved and the qualifications of the movement. It would thus appear that the Isochrony Principle is a common emerging feature of otherwise inependent motor systems, rather than one specific component shared by all these mechanisms. It follows that, for any given motor performance, one should ideally be able to show how and why the specific logic and constraints of the relevant motor mechanisms result in such compensatory behavior.

In this and in another paper ${ }^{7}$ we take up such a problem for a class of simple hand and finger movements. The motor task considered is the spontaneous drawing of closed patterns: circles, ellipses and several forms of the "Figure Eight". Despite their simplicity, these movements have a considerable interest insofar as they are the building blocks for such highly skilled motor performances as handwriting and drawing.

Previous work on handwriting ${ }^{6,11,13}$ has described some of the principles of organisation of writing movements. It was shown that the kinematics of writing is highly correlated with movement trajectory and, in particular, that to each word written in a given size corresponds a unique profile of tangential

*Present address: Istituto Fisiologia Centri Nervosi, CNR, via Mario Bianco, I-20131 Milano, Italy.

†Present address: Neurological Science Institute, Good Samaritan Hospital \& Medical Center, Portland, Oregon, U.S.A. velocity. When the same word is written in different sizes, the shape of the velocity profile remains the same, but the average velocity changes pari passu with the size so that execution time tends to remain constant. Thus, even in the case of complex motor sequences involving curvilinear trajectories and reversals of direction, the velocity of the movement is related-via the Isochrony Principle - to total linear extent of the trajectory. ${ }^{2}$ Instead, when a closed pattern is traced repeatedly, as in the experiments to be described here, the Principle seems to apply to the linear extent of just one cycle of the movement rather than to the total length of the trace. ${ }^{7}$

With respect to the examples of monodimensional movements mentioned above, for which only the extent appear to have a major effect on the kinematics, the case of bidimensional writing-like movements is considerably more complex, inasmuch as the instantaneous velocity also depends on the local geometrical properties of the trajectories. All (but one) of the experiments to be reported were primarily designed to single out the dependence of the average velocity on the global metric properties of the trajectory for a class of patterns with constant curvature. The dependence of the instantaneous velocity on the local geometrical properties of the trajectory is dealt with in a separate paper. ${ }^{6}$

\section{EXPERIMENTAL PROCEDURES}

Horizontal and vertical components of the drawing movements were recorded with a Calcomp 622 RP digitizing table (accuracy $0.025 \mathrm{~mm}$; sampling rate $100 \mathrm{~Hz}$ ). The writing stylus provided with this table closely resembles an ordinary ball pen. The displacement of the tip of the pen could be measured reliably, also when the pen was not touching the paper, as long as it stayed within $1 \mathrm{~cm}$ from the table. Time derivatives of the movement components were calculated numerically with a 5-point polynomial formula after smoothing the raw data with a double-sided, numerical low-pass filter (cut-off $50 \mathrm{~Hz}$ ). Curvature was calculated from these time derivatives. All the patterns to be drawn were closed (see below). The task consisted in tracing these patterns repeatedly, at a spontaneously selected speed. In all 
cases the experimenter let the movement go for a few cycles before recording for $10 \mathrm{~s}(1000$ samples at the $100 \mathrm{~Hz}$ sampling rate). Four subjects (two paid students and the two authors) participated in the experiments. The instructions for the various experimental conditions were given verbally in the course of an informal practice session.

\section{RESULTS}

Figure 1 shows typical examples of the recorded quantities for two of the patterns to be considered. Each panel in this Figure illustrates the time course of the tangential velocity $V$ and of the radius of curvature $R$ for the pattern indicated. As already demonstrated in a previous report, ${ }^{13}$ a striking correlation exists between the instantaneous values $V(t)$ and $R(t)$. With the exception of the inflection points, where $R$ goes to infinity (see upper panel), the proportional relation $V(t)=k R(t)$ provides a first order approximation to the data. In what follows, we will mostly be concerned with the relation between the average values of the tangential velocity $V$ and of the radius $R$ (or, equivalently, the curvature $C=1 / R$ ) over identifiable figural units. Thus, for instance, in the case of the patterns shown in Fig. 1, the averages of $V$ and $R$ were independently calculated over the two circles which form the pattern and which obviously represent distinct figural units (not necessarily distinct units of motor action; see ref. 12).

The first experiment deals with the drawing of circles. Subjects were to draw successively a number of circles with varying diameters. The exact value of each diameter, as well as the sequence of these values, was left to each subject's whim. We gave, however, a rough indication of the extreme values to be attained, and we suggested that the entire range be covered in an approximately uniform manner. Three of the subjects wre left free to choose the direction of the movement which was kept constant throughout the experiment. A fourth subject performed the experiment in both directions. All subjects used their dominant hand. Figure 2 summarizes the results. For the sake of clarity, only the results of three subjects are shown. In A, different symbols describe the relation between average curvature and average velocity for clockwise (O) and counterclockwise (O) movements, in one subject. In B, each symbol identifies onc of the
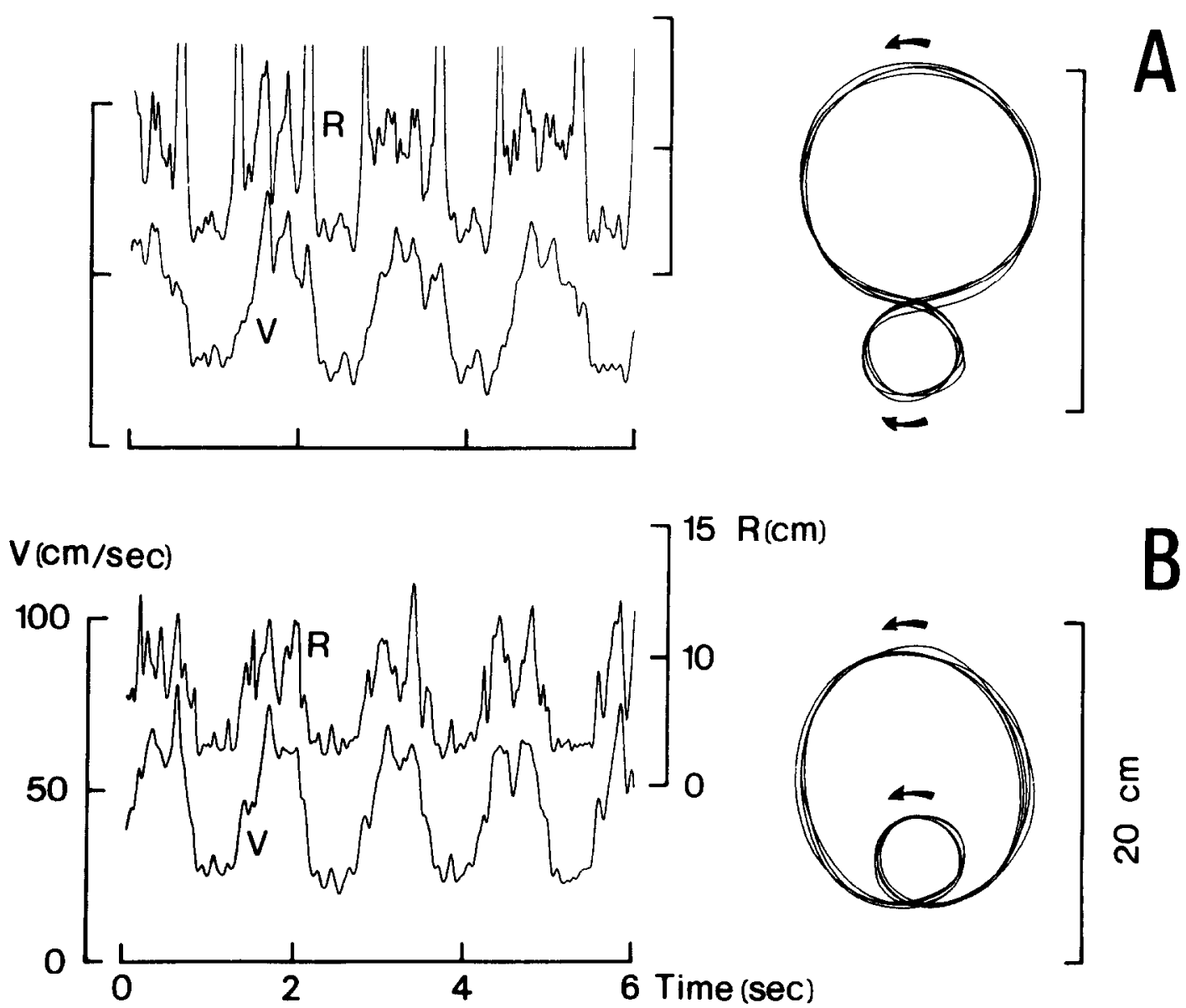

Fig. 1. Kinematic description of the movement for two typical examples. In $A$ and $B$ are shown two of the patterns considered in this study and the corresponding time course of the radius of curvature (R) and of the modulus of tangential velocity (V). Notice the close correlation between these two variables. 


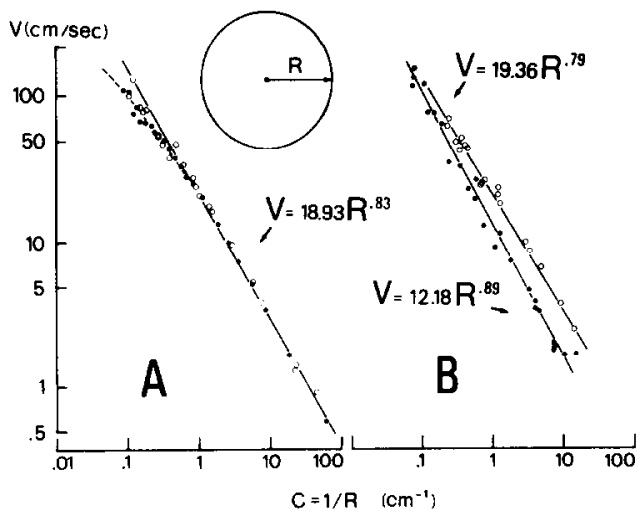

Fig. 2. Relation between the average tangential velocity and the average radius of curvature in the case of circles. A: Results in one subject for clockwise (O) and counterclockwise $(O)$ movements. B: Results for two other subjects identified by different symbols (clockwise movements). The straight line interpolations correspond to the indicated power functions. In circles, the radius of curvature is proportional to the total linear extent (perimeter) of the pattern.

other two subjects. The straight lines interpolating the data points correspond to the indicated power functions. Apart from a small but noticeable curvature in the upper part of the curve in A, the approximation of the results by power functions is extremely good. Thus, in a range of movement sizes going from those that involve the elbow and the shoulder, to almost point-like ones, and whatever the direction of the movement, a simple relation exists between the radius of curvature (or, equivalently, the length of the trajectory) and the speed of execution:

$$
V=K R^{\mathrm{a}} \text {. }
$$

Notice that the exponent of the power function for all subjects is significantly less than 1 . Therefore, the time to draw a circle is a slightly increasing function of the circle size: isochrony is only partially attained.

The remaining experiments wcrc designed to investigate the extent to which the relation demonstrated for circles generalizes to more complex patterns. The instructions and modalities were similar to those of the first experiment. However, in order to minimize inter-individual variability, we prepared for each experiment a set of templates of different sizes, drawn lightly on paper, which served as a guide for the first few cycles of the movement (see Experimental Procedures). It should be stressed that, by the time the recording started, the templates were abundantly written over and therefore virtually invisible: during the recording the subjects were actually copying their previous movements as in the case of circles.

Figure 3 shows the results in two subjects ( $\mathrm{A}$ and B) for the Figure Eight pattern with circles of equal size. In both panels of this Figure, different symbols identify the relationship between the averages of $R$ and $V$ for each of the two halves of the pattern. In
B the two sets of data points permit comparison of the performances of the same subject at a few days of interval. Continuous lines represent the best-fitting power functions for the data. Dashed lines reproduce, for each subject, the fitting to the circle data already shown in Fig. 2. The average velocities for the Figure Eight pattern are typically smaller than those for circles. However, the same type of relation, with similar values for the exponents, holds in both cases. From this we conclude that, despite the alternations from clockwise to counterclockwise rotations in these movements, the principle of organisation valid for simple units (circles) remains valid also when two such units are coordinated in one, more complex pattern.

As a further generalisation, we consider the drawing of a Figure Eight with unequal radii. A typical result of this experiment has been shown in Fig. 1A, while the complete results are summarized in Fig. 4. Experiments were performed in three successive sessions. In each session the radius of the upper circle $\left(R_{1}\right)$ was kept constant, while that of the lower one $\left(R_{2}\right)$ was varied. In all thrce cases, the range of variation of $R_{2}$ included the (fixed) value of $R_{1}$. Thus, the experiments replicated three measurements of the previous one. In Fig. 4, the average values of the tangential velocity for the two parts of the Figure $(O$ : $V_{1}, 0: V_{2}$ ) are plotted as a function of the average radius of curvature $R_{2}$ (which can also be expressed as curvature $C_{2}$ ). The results show unambiguously that:

(1) The average velocities $V_{1}$ and $V_{2}$ in the two parts of the pattern differ considerably;

(2) The $V_{2}-R_{2}$ relationship for the circle with variable radius is no longer a simple power function as in the previous experiments;

(3) The velocity $V_{1}$ in the circle with fixed radius not only depends on $R_{1}$ but on $R_{2}$ as well.

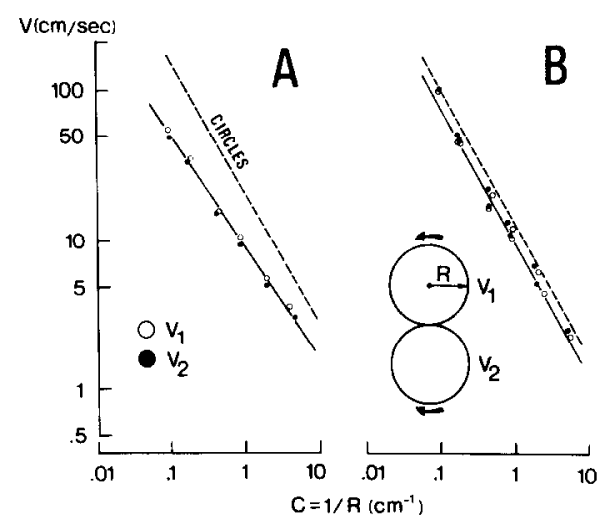

Fig. 3. Symmetrical Figure Eight. Results in two subjects (A and $\mathrm{B}$ ). Different symbols describe the $V-R$ relationship in the two parts of the patterns. Dashed lines indicate the power function fits to the circle data for the same subjects (cf Fig. 2). As in circles, the radius of curvature is proportional to the perimeter of the pattern. 


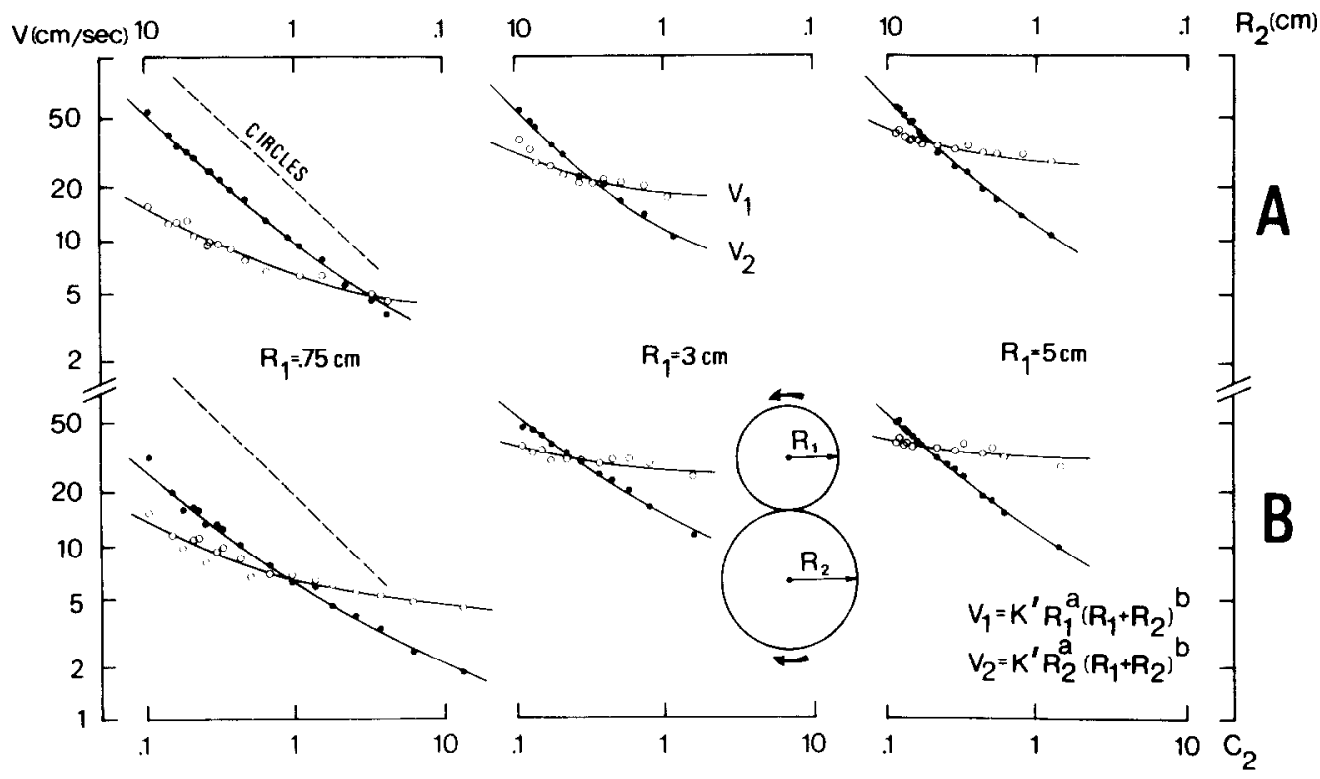

Fig. 4. Asymmetrical Figure Eight. Results in two subjects (A and B). Different symbols describe the $V-R_{2}$ relationship in two parts of the patterns for each of the indicated values of the radius of the upper circle. Dashed lines indicate the power-function fits to the circle data for the same subjects (see Fig. 2). Notice that neither $V_{1}-R_{2}$ nor the $V_{1}-R_{2}$ relations are adequately described by simple power functions. The continuous lines, which provide an excellent interpolation of the data points, correspond to the double power functions reported inset.

Considering that, in the case of circles, the length of the trajectory is proportional to the radius of curvature, points 2 and 3 above can be tentatively summarized by saying that the average velocity in each part of the pattern depends on both the length of that part and the length of entire pattern (see, however, Discussion). This conclusion can be formulated quantitatively by assuming that the constant $\mathrm{K}$ in equation (1) is itself a power function of the total length:

$$
\begin{aligned}
& V_{1}=K^{\prime} R_{1}^{\mathrm{a}}\left(R_{1}+R_{2}\right)^{\mathrm{b}} \\
& V_{2}=K^{\prime} R_{2}^{\mathrm{a}}\left(R_{1}+R_{2}\right)^{\mathrm{b}} .
\end{aligned}
$$

The best fitting approximation of Equation (2) to the data points (continuous lines in Fig. 4) is indeed excellent. The corresponding estimates of the coefficient $K^{\prime}$, and of the exponents a and b (Table
1) are consistent with those of the first two experiments. Indeed, in the case of Figure Eight pattern with equal radii, Equation (2) reduces to:

$$
V=2^{\mathrm{b}} K^{\prime} R^{(\mathrm{a}+\mathrm{b})}
$$

and the average sum $a+b$ of the estimated exponents is numerically close to the exponents of the best fitting power functions in Figs 2 and 3.

The identification of the sum $R_{1}+R_{2}$ with the length of the trajectory is reasonable but not logically inescapable. Because of the special properties of the Figure Eight, it is also possible to identify $R_{1}+R_{2}$ with the overall vertical extent of the movement. In order to climinate this ambiguity, and further to extend validity of the results, we considered the drawing of a "folded-up" version of the Figure Eight

\begin{tabular}{|c|c|c|c|c|c|c|c|}
\hline \multirow[b]{2}{*}{$R_{1}$} & \multicolumn{3}{|c|}{$S_{1}$} & \multicolumn{3}{|c|}{$\mathrm{S}_{2}$} & \\
\hline & $\log K^{\prime}$ & a & b & $\log K^{\prime}$ & a & $b$ & \\
\hline 0.75 & 1.85 & 0.40 & 0.50 & 1.59 & 0.30 & 0.42 & \\
\hline 3.00 & 1.98 & 0.40 & 0.50 & 2.40 & 0.44 & 0.25 & Asymmetrical \\
\hline 5.00 & 2.00 & 0.52 & 0.40 & 2.07 & 0.58 & 0.25 & Figure-of-Eight \\
\hline Av. & 1.94 & 0.44 & 0.47 & 2.02 & 0.44 & 0.31 & \\
\hline$R_{1}$ & $\log K^{\prime}$ & a & b & $\log K^{\prime}$ & a & b & \\
\hline 3.00 & 1.87 & 0.61 & 0.43 & 2.68 & 0.63 & 0.21 & Folded \\
\hline 8.00 & 0.39 & 0.60 & 0.99 & 2.31 & 0.64 & 0.57 & Figure-of-Eight \\
\hline
\end{tabular}
pattern, shown diagrammatically in Fig. 5 (Fig. 1B

Table 1. Best fitting parameters

Values of the parameters $K^{\prime}$, a and b in Equation 2 which produce the interpolations shown in Figs 4 and 5 . Results in two subjects $\left(S_{1}\right.$ and $\left.S_{2}\right)$. 

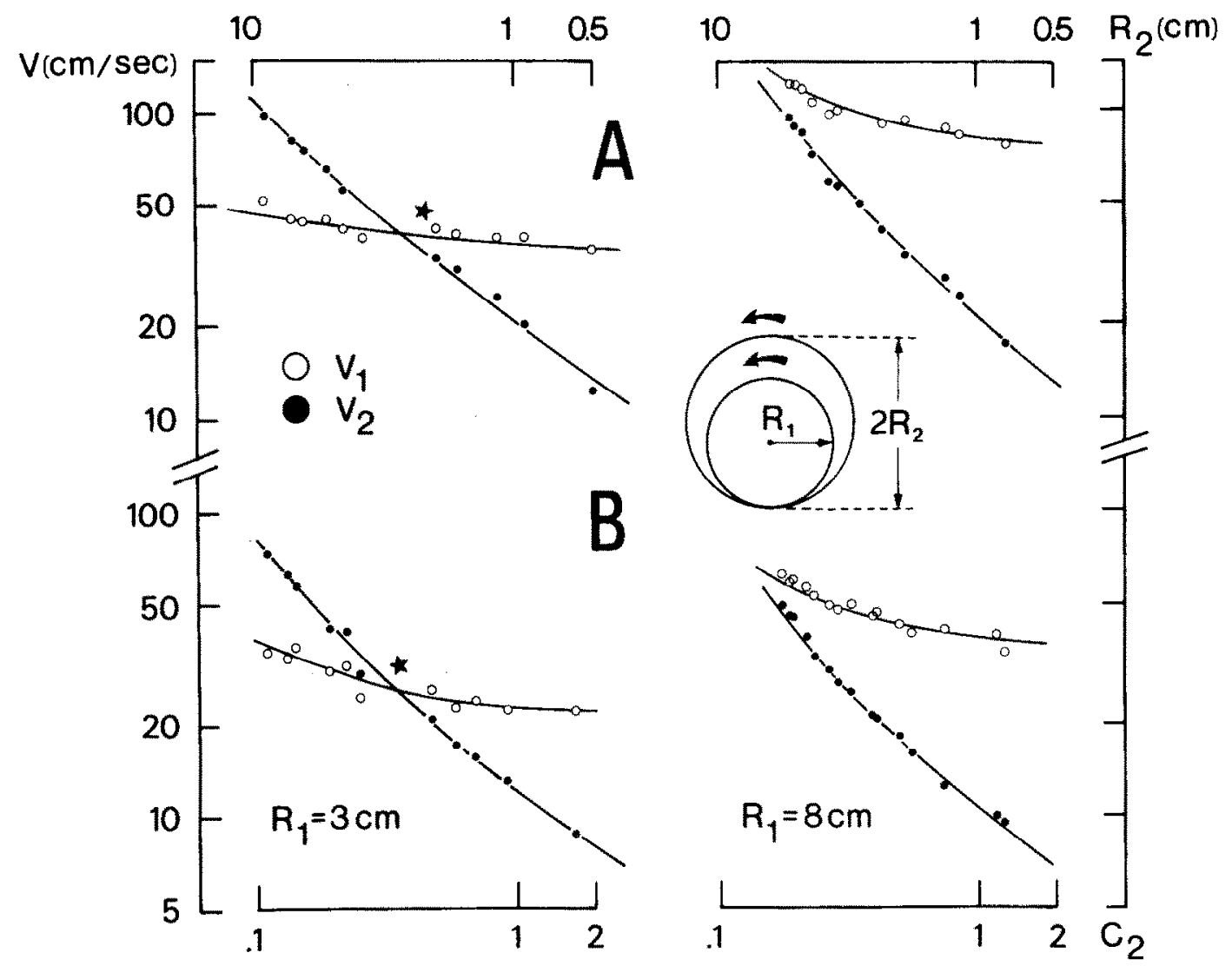

Fig. 5. Folded-up Figure Eight. Results in two subjects (A and B). The radius of one circle is kept constant, the other ranges between 0.75 and $7 \mathrm{~cm}$. When $R_{1}=3 \mathrm{~cm}$ (left panel), the fixed-sized circle is either inside or outside the variable one, depending on the radius of the latter. When $R_{2}=8 \mathrm{~cm}$, the variable circle is always smaller than the constant one and, therefore, the $V-R$ characteristics do not cross. The data points $(\star)$ correspond to the case $V_{1}=V_{2}=3$ in which the pattern becomes a circle.

shows an actual measurement for this type of pattern). Experiments were run in two successive sessions. In each session one radius (conventionally referred to as $R_{1}$ ) was kept constant while the other $\left(R_{2}\right)$ was varied. When $R_{1}=8$ (right panels in Fig. 5), the overall vertical extent of the movement $(16 \mathrm{~cm})$ is independent of $R_{2}$, while the length of the trajectory is still proportional to $R_{1}+R_{2}$ as in the previous experiments. In this case $R_{2}$ was always smaller than $R_{1}$. Thus, $V_{2}$ is always less than $V_{1}$ and the $V_{1}-R_{2}$ and $V_{2}-R_{2}$ characteristics do not cross as they do in Fig. 4. However, the results in two subjects demonstrate that even in this case Equation (2) provides a very satisfactory interpretation of the results. It is therefore confirmed that the average velocity in each part of these patterns is expressible by two power functions components, one of which can be identified with the total pattern length. However, the estimates of the parameters $a$ and $b$ (Table 1) are significantly different from those calculated previously. The control condition for this experiment is provided by the case $R_{1}=3$ (left panels in Fig. 5). Here the range of $R_{2}$ includes the fixed value of $R_{1}$.

Thus, apart from the fact that no inversion of movement direction occurs in the drawing of this pattern, the conditions are the same as in the regular Figure Eight experiment (Fig. 4). For both subjects, the results are very similar to those for $R_{1}=8$ and so are the estimates of the parameters. Notice that when $R_{1}=R_{2}=3$; the pattern collapses into a circle. The corresponding average velocity (data points $\star$ ) is slightly but characteristically higher than that predicted by the crossing of the theoretical power functions. This suggests that the very presence of two distinguishable units in the pattern has an influence in the overall dynamic organization of the movement. From the results of both experiments we conclude that switching from one direction of rotation to the opposite one does not modify the form of the $R-V$ relationship. However, the fact that an inversion is, or is not, present may be responsible for the difference in the exponents.

To conclude, we shall consider the drawing of patterns with regularly changing curvature. The templates (outlined on paper as in the other experiments) were two isoperimetric ellipses with a high $(-0.95)$ and low $(O=0.60)$ eccentricity, respectively. Each template was drawn in nine different sizes arranged in 
a geometric progression and ranging from 36 to $573 \mathrm{~mm}$ in perimeter. It was shown previously, ${ }^{13}$ that in the drawing of ellipses the angular velocity oscillates rather abruptly between two distinct values. These transitions occur approximately at the four symmetrical points of the trajectory where the radius of curvature is the arithmetic mean of its maximum and minimum values. Since each cycle of the movement can be thus divided into two pairs of symmetrical portions within which the angular velocity is relatively constant, we averaged the instantaneous values of $V$ and $R$ over each pair independently. For a given eccentricity and a given size, such procedure yields two points in the $V-R$ plane, one for the poles and the other for the flatter parts of the contour. When the size of the ellipsis decreases, the average values of the curvature $(C=1 / R)$ increase, while the corresponding average values of the velocity $V$ decrease as in Fig. 2-5. Thus, spanning the entire range of sizes yields two distinct $V-R$ characteristics for each eccentricity. Typical results in one subject are illustrated in Fig. 6. They show that all four $V-R$ curves have approximately the same slope as those for the circle in the same subject (broken line). Thus, the relation between length and velocity of execution which has been demonstrated in patterns with constant curvature is also valid when a systematic variation of curvature is present.
During the execution of any given ellipsis the instantaneous values of the angular velocity, and of the closely related tangential velocity, change continuously as a function of the instantaneous values of the curvature. More specifically, it can be shown ${ }^{6}$ also that the instantaneous values of $V$ and $R$ are related by a power law. An approximation to this relationship for any given ellipsis can be here provided by joining with linear segments the corresponding average points. This is shown in Fig. 6 only for the more eccentric ellipsis (dashed lines). The slope of these linear segments (i.e. the exponent of the power law) is similar for all sizes. The average slope is suggested by the dot-dashed line which represents the power function $V^{\prime}=K R^{0.27}$.

\section{DISCUSSION}

In writing and drawing movements, the instantaneous tangential velocity of the pen's tip is a function of both the global parameters of the trajectory and of its local (differential) properties. In the examples of Fig. 1, the effects of the local parameters show up in the close similarity between the high frequency oscillations of the curvature $R(t)$ and the corresponding values of the tangential velocity $V(t)$. These effects, already described in Viviani and Terzuolo ${ }^{13}$, are considered in detail in a separate paper." In the

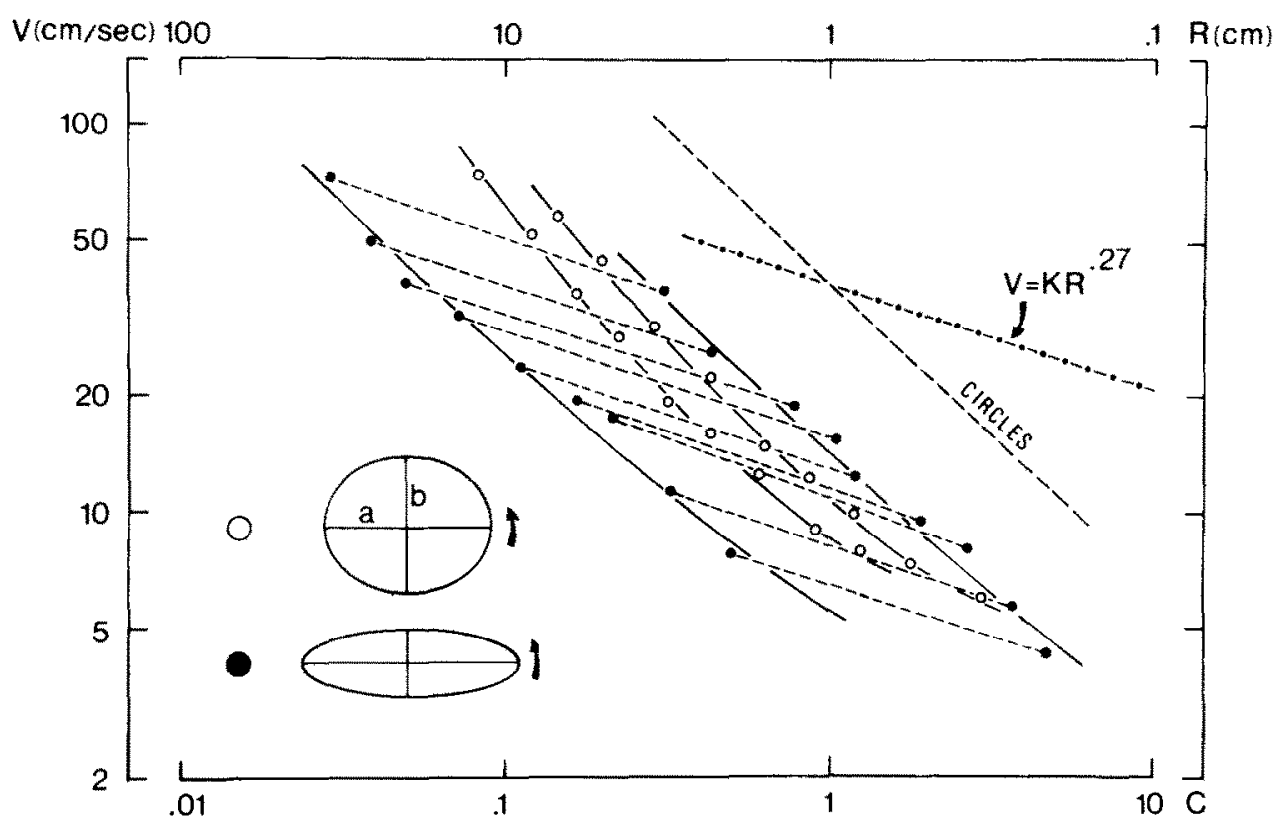

Fig. 6. Ellipsis. Data in one subject. Different symbols describe the $V-R$ relation for ellipses with high (O) and low (O) eccentricity. For each ellipsis two $V-R$ characteristics are plotted, one for portion of trajectory where $R$ is higher than the mean value of the radius (poles), the other for the flatter portions, where $R$ is smaller than the mean value. All four $V-R$ characteristics have approximately the same slope as the circle data for the same subject (cf, dashed line labelled "circles"). The dashed segments which connect each pair of corresponding points on the two characteristics are drawn to suggest the relation between the values of the tangential velocity and of the radius of curvature within each cllipsis. The average slope of the dashed segments is suggested by the dot-dashed line which represents the power function $V=K R^{0.27}$. 
experiments presented here, we have instead sought to isolate the effects of the global parameters, by considering closed patterns with either constant or regularly changing curvature.

The fundamental hypothesis of the study is that the high-frequency, local effects can be "factored out" from the raw data by averaging velocity and curvature over identifiable segments of the trajectory (such as the two circles which compose the Figure Eight). The residual variations of velocity would then represent a "Gain" factor which can be assumed to remain constant over those segments and which only depends on the global parameters of the trajectory (cf. ref. 7). With reference to the examples of Fig. 1, our hypothesis leads us to assume that useful information on this gain factor can be obtained by approximating $V(t)$ with piece-wise constant functions.

In the case of circles (Fig. 2) and symmetrical Figure Eights (Fig. 3), the average radius of curvature of the trajectory is proportional to its total length. Therefore, the very little scatter of the data points and their excellent interpolation by Equation (1) suggest the existence of a very accurate gain control mechanism relating the average tangential velocity and the linear extent of one cycle of the movement. To be sure, this relation is not rigid inasmuch as we can certainly draw intentionally the same circle at two different speeds. Nevertheless, there seems to exist a preferred velocity which each individual spontaneously selects when he/she performs a movement of a given size. This velocity may differ (appreciably) from subject to subject and (slightly) from one experiment to the next (cf. panel B in Fig. 3). However, when one velocity value has been selected for one size, the drawing of the same pattern in other sizes is constained by a tight covariation between speed and extent which is reminiscent of the Isochrony Principle ${ }^{2.5 .10}$ and which can be interpreted as the operating characteristic of the individual performances.

The experiments with asymmetrical (Fig. 4) and folded (Fig. 5) Figure Eights further qualify the dependency of the gain factor from the global metric properties of the trajectory. The large and systematic changes observed in different portions of the movement are in fact incompatible with the hypothesis that the average tangential velocity only depends on the perimeter of the pattern. The representation of the results by Equation (2) actually proves that the average tangential velocity can be factored out into at least two multiplicative components. One component can be reliably identified with the total perimeter of the pattern. As for the other component, good interpolation of the data provided by Equation 2 indicates that it depends on the radii of the two subpatterns that form the movement. Thus, a possible interpretation of Equation 2 is that the average velocity in each identifiable subpattern of a complex movement depends on both the total perimeter of the pattern and the perimeter of that subpattern. The ellipsis experiments (Fig. 6) suggests, however, a different interpretation which is in better agreement with previous results. ${ }^{13}$ It demonstrates, in fact, an effect of the radius of curvature, which is independent of the perimeter of the pattern. If we assume that this specific effect is also present in all other cases considered so far, where, however, it cannot be distinguished from that of the length of the trajectory, we may then tentatively identify the second multiplicative component of the tangential velocity with the average radius of curvature. This hypothesis is in keeping with the quantitative analysis of the data. Indeed, in the case of the ellipsis also, the tangential velocity in each portion of the trajectory can be expressed as the product of two terms:

$$
V=K^{\prime} R^{0.30} P^{b}
$$

where $R$ is the average radius of curvature of that portion, and $P$ is the perimeter of the ellipsis. When the eccentricity tends to zero, $P$ tends to $2 \pi R$ while the sum of the exponents $0.30+b$ should coincide with the average estimated exponent for the circle, say 0.80 (cf. Fig. 2). If, as a first approximation, we assume that this relation between exponents also applies for non-zero eccentricities we obtain $b=0.50$, that is:

$$
V=K^{\prime} R^{0.30} P^{0.50}
$$

These estimates are not very different from those of the exponents $a$ and $b$ in Equation (2) for the asymmetrical Figure Eight (Table 1) which, as already noted, are themselves coherent with the results for circles and symmetrical Figure Eights. The "a" exponents for the folded patterns are instead higher $(\sim 0.60)$ while the $b$ exponents are somewhat variable. Thus, although the changes in movement direction necessary to draw these patterns seem to have a specific quantitative effect on the timing of the movement, one and the same qualitative law relating velocity, curvature and perimeter applies to all closed patterns considered in these experiments.

To conclude, we would like to raise a theoretical issue concerning the general organisation of the motor plan. Whenever a given closed pattern is repeated over and over as in our experiments, it is quite natural to assume (cf. Introduction) that the larger linear extent relevant to the planning of the movement is the perimeter of the pattern. More specifically, it is natural to assume that the gain factor which regulates the average speed of execution only depends on the perimeter of the planned trajectory, being instead unaffected by the additional instruction to execute so and so many times the same plan. Informal observations have indeed shown that the tempo of the movement is practically independent of the number of repetitions. However, periodic, closed movements, are but a special case of all possible smooth movements. On an a priori basis, it seems unlikely that such a sophisticated principle of planning as the precise dependency of the velocity from 
the linear extent has developed only for such special case. It is then tempting to speculate that some notion of linear extent must be represented internally also in the general case of open, aperiodic movements. If this is true, one could further speculate that such generalized notion of linear extent applies to those successive segments of the trajectory whose identification is relevant to the global motor plan (cf. ref. 7). Ultimately, it is conceivable that these segments, if they exist, might be identified by a systematic analysis of the correlation between the extent of all possible stretches of the trajectory and the corresponding average velocity of execution.

Acknowledgements - - This research was partly supported by ATP-INSERM Research Grant A 650-5169 to P.V. Dr McCullom was supported by a Visiting Scientist Fellowship by INSERM.

\section{REFERENCES}

1. Binet A. and Courtier J. (1893) Sur la vitesse des mouvements graphiques. Revue Phil. 35, 664-671.

2. Braitenberg B. (1965) A note on the control of voluntary movements. In Cybernetics of Neural Processes (ed. Caianiello E. R.) C.N.R., Roma.

3. Bryan W. L. (1892) On the development of voluntary motor ability. Am. J. Psychol. 5, 125-204.

4. Fitts P. M. (1954) The information capacity of the human motor system in controlling the amplitude of the movement. J. exp. Psychol, 47, 381-391.

5. Freeman F. N. (1914) Experimental analysis of the writing movement. Psychol Rev. Monograph. Suppl. $17,1-46$.

6. Lacquaniti F., Terzuolo C. A. and Viviani P. (1983) The law relating the kinematic and figural aspects of drawing movements. Acta Psychol. In press.

7. Lacquaniti F., Terzuolo C. A. and Viviani P. (1983) Global metric properties and preparatory processes in drawing movements. In Preparatory States and Processes (eds Kornblum S. and Requin J.) In press.

8. Michel F. (1971) Etude expérimentale de la vitesse du geste graphique. Neuropsychologie 9, 1-13.

9. Stark L. (1968) Neurological Control Systems, Siudies in Bioengineering. Plenum Press, New York.

10. Stetson R. H. and McDill J. A. (1923) Mechanism of the different types of movement. Psychol. Monograph 32, 18-40.

11. Viviani P. and Terzuolo C. A. (1980) Space-time invariance in learned motor skill. In Tutorials in Motor Behavior (eds Stelmach E. G. and Requin J.), pp. 525-533. North-Holland, Amsterdam.

12. Viviani P. and Terzuolo C. A. (1983) The organization of movement in handwriting and typing. In The Production of Language in Non-Speech Modalities (ed. Butterworth B.) Academic Press, London. In press.

13. Viviani P. and Terzuolo C. A. (1982) Trajectory determines movement dynamics. Neuroscience 7, 431-437.

14. Zangemeister W. H., Lehman S. and Stark L. (1981a) Simulation of head movement trajectories: model and fit to main sequence. Biol. Cybernetics 41, 19-32.

15. Zangemeister W. H., Lehman S. and Stark L. (1981b) Sensitivity analysis and optimization for a head movement model. Biol. Cybern. 41, 33-45.

16. Yarbus A. L. (1967) Eye Movements and Vision. Plenum Press, New York. 